\title{
Phosphorylation of PRAS40 contributes to the activation of the PI3K/AKT/mTOR signaling pathway and the inhibition of autophagy following status epilepticus in rats
}

\author{
JUNYI LIN ${ }^{1,2 *}$ YOUXIN FANG ${ }^{3 *}$, MINGCHANG ZHANG ${ }^{2 *}$, XIANG WANG ${ }^{4}$, LILIANG LI $^{2}$, \\ MENG HE ${ }^{2}$, AIMIN XUE ${ }^{2}$, KEMING ZHU ${ }^{5}$, YIWEN SHEN ${ }^{2}$ and BEIXU LI ${ }^{1,2}$ \\ ${ }^{1}$ School of Policing Studies, Shanghai University of Political Science and Law, Shanghai 201701; \\ ${ }^{2}$ Department of Forensic Medicine, School of Basic Medical Sciences, Fudan University, Shanghai 200032; \\ ${ }^{3}$ General Family Medicine Clinic, Xuhui District Xietu Subdistrict Community Healthcare Center, Shanghai 200120; \\ ${ }^{4}$ Institute of Forensic Science, Jiading Branch of Shanghai Public Security Bureau, Shanghai 201800; ${ }^{5}$ Department of Anatomy, \\ Histology and Embryology, School of Basic Medical Sciences, Fudan University, Shanghai 200032, P.R. China
}

Received October 7, 2018; Accepted June 2, 2020

DOI: $10.3892 /$ etm.2020.9085

\begin{abstract}
Status epilepticus (SE) is a neurological disorder associated with high morbidity and mortality rates, and is often difficult to treat. Moreover, the underlying mechanism of SE remains unknown. The lithium-pilocarpine model is a validated animal model that can reproduce the main clinical and neuropathological features of SE. In the present study, this SE model was utilized and SE was successfully established in rats, as determined by the corresponding epileptic electroencephalogram. Histology, immunohistochemistry, western blot analysis and co-immunoprecipitation were used to detect the phosphorylation (p-) of AKT substrate of $40 \mathrm{kDa}$ (PRAS40), the combination of p-PRAS40 and 14-3-3 protein and the activation of the PI3K/mTOR signaling pathway in SE. In addition, the present study analyzed the dynamics of the expression of autophagy-associated factors in the hippocampus after SE induction, and the influence of suppressing
\end{abstract}

Correspondence to: Dr Beixu Li, School of Policing Studies, Shanghai University of Political Science and Law, 7989 Waiqingsong Road, Shanghai 201701, P.R. China

E-mail: libeixu@fudan.edu.cn

Professor Yiwen Shen, Department of Forensic Medicine, School of Basic Medical Sciences, Fudan University, 131 Dongan Road, Shanghai 200032, P.R. China

E-mail: shenyiwen@fudan.edu.cn

*Contributed equally

Abbreviations: SE, status epilepticus; PRAS40, proline-rich AKT substrate of $40 \mathrm{kDa}$; P70S6K, ribosomal protein S6 kinase 1; IHC, immunohistochemistry; EEG, electroencephalography; MFS, Mossy-fiber sprouting

Key words: SE, mTOR pathway, phosphorylated-PRAS40, autophagy the p- of PRAS40 on the autophagy process was detected in the pathogenesis of SE. The results indicated that increased p-PRAS40 expression could activate the mTOR pathway to decrease the level of autophagy. However, inhibition of the mTOR signaling pathway promoted autophagy flux. These results may provide further understanding of p-PRAS40 functions in SE.

\section{Introduction}

Status epilepticus (SE) is one of the most common types of neurological disorders, and can cause patient disability or mortality if not diagnosed and treated early (1). SE can have long-term consequences following $30 \mathrm{~min}$ (tonic-clonic SE) or $60 \mathrm{~min}$ (focal SE with impaired consciousness), including neuronal death, neuronal injury and alteration of neuronal networks (2). However, the underlying mechanism of SE is yet to be elucidated.

The mTOR signaling pathway is an important signaling mechanism in epileptogenic processes (3). AKT substrate of $40 \mathrm{kDa}$ (PRAS40) is a novel downstream factor of the PI3K/AKT signaling pathway and was first identified by Kovacina et al (4) in 2003. When the PI3K/AKT signaling pathway is activated, PRAS40 can be phosphorylated (p-) by p-AKT at Thr246 site and promote the signal downstream to the mTOR signaling pathway (5). Moreover, PRAS40 is a subunit of the mTOR complex 1 (mTORC1). PRAS40 inhibits mTORC1 autophosphorylation (6) and prevents mTORC1 binding to downstream ribosomal protein S6 kinase 1 (P70S6K) and eukaryotic initiation factor $4 \mathrm{E}$ binding protein 1 (7), in order to inhibit the activation of the mTOR signaling pathway. In addition, p-PRAS40 binds to the 14-3-3 scaffold protein and separates from mTORC1, and does not inhibit the mTOR signaling pathway (8). PRAS40 also participates in the mTOR signaling pathway by regulating neural development, circuit formation and synaptic plasticity (9).

Abnormal activation of the mTOR pathway in SE has been reported by previous studies. For instance, San et al (10) revealed 
the elevation of the p-mTOR/mTOR and p-P70S6K/P70S6K ratios in the posttraumatic amnesia-induced SE rat model. Furthermore, Brewster et al (11) showed that mTORC1 hyperactivation was associated with SE-induced memory deficits and dendritic dysregulation, which could be partially reversed by rapamycin, an inhibitor of the mTOR pathway. In a study by Wang et al (12), it was demonstrated that hyperactivation of the mTOR pathway could lead to increased levels of NF- $\mathrm{KB}$, as well as the promotion of inflammation in the brain early after SE. However, to the best of our knowledge, there have been few reports focused on the association between PRAS40 and SE.

Autophagy is one of the most important methods to eliminate and recycle intracellular materials in eukaryotic cells (13). Under conditions of nutrient sufficiency, the mTOR pathway is activated when mTORC1 inhibits autophagy by phosphorylating the Unc-51 like autophagy activating kinase 1 complex, which is a promoter of autophagy (14). However, under nutrient starvation, opposite events lead to the elevation of autophagy levels and cytoplasmic contents are eliminated to generate energy for essential cellular activities (10). There has been controversy regarding autophagy-associated alterations following SE. For example, some previous studies reported an abnormal elevation in autophagy levels following SE $(15,16)$, but other researchers have hypothesized that autophagy-associated activities induce dynamic changes during this cellular event (17-19). As mTOR is one of major pathways that regulates the onset of autophagy, it was hypothesized that PRAS40 may participate in this regulation by influencing the activity of the mTOR signaling pathway.

The aim of the present study was to investigate the role of PRAS40 in SE and the associated mechanism, as well as how PRAS40 participates in the mTOR associated regulation of autophagy following SE.

\section{Materials and methods}

Antibody and reagents. Primary antibodies were purchased as follows: P-PRAS40 (Thr246; cat. no. 2997; Cell Signaling Technology, Inc.), PRAS40 (cat. no. 2691; Cell Signaling Technology, Inc.), p-mTOR (Ser2448; cat. no. 5536; Cell Signaling Technology, Inc.), mTOR (cat. no. 2983; Cell Signaling Technology, Inc.), p-AKT (Ser473; cat. no. 4060; Cell Signaling Technology, Inc.), AKT (cat. no. 4685; Cell Signaling Technology, Inc.), p-P70S6K (Thr389; cat. no. 9234; Cell Signaling Technology, Inc.), P70S6K (cat. no. 14130; Cell Signaling Technology, Inc.), Light chain 3-I/II (LC3-I/II; cat. no. 12741; Cell Signaling Technology, Inc.), 14-3-3 (cat. no. 8312; Cell Signaling Technology, Inc.), P62 (cat. no. 18420-1-AP; ProteinTech Group, Inc.) and GAPDH (cat. no. sc-365062; 1:2,000; Santa Cruz Biotechnology, Inc.). Goat anti-rabbit horseradish peroxidase (HRP)-conjugated secondary antibody (cat.no. sc-2054; Santa Cruz Biotechnology, Inc.). The following drugs were obtained from commercial sources as follows: Pilocarpine (cat. no. S4231; Selleck Chemicals), lithium chloride (cat. no. L9650; Sigma-Aldrich; Merck KGaA), Scopolamine (cat. no. S2508; Selleck Chemicals) and LY3023414 (cat. no. S8322; Selleck Chemicals).

Animals and drug treatment. 50 adult male Sprague Dawley rats which provided by Shanghai SLAC Laboratory Animal
Co. Ltd. (weight, 200-250 g; age, 5 weeks old) were used in the present study and the protocols used were approved by the Experimental Animal Ethics Committee of the Basic Medical College of Fudan University (approval no. 20170223-066). Rats were housed in standard cages with free access to food and water on a 12-h light/dark cycle in a temperature-controlled room $\left(26^{\circ} \mathrm{C}\right.$, the humidity was $\left.40-60 \%\right)$ and were allowed a 3 days period to acclimatize prior to treatment with drugs. Lithium chloride [127 mg/kg; intraperitoneal (i.p.)] was administered $18 \mathrm{~h}$ prior to pilocarpine administration. Pilocarpine (30 mg/kg; i.p.) was administered $1 \mathrm{~h}$ following pre-treatment with scopolamine $(1 \mathrm{mg} / \mathrm{kg}$; i.p.; to prevent the effects of peripheral cholinergic stimulation) to induce seizures. The electroencephalograms (EEG) was acquired and analyzed with a RM6240C multichannel physiological signal acquisition and processing system (Chengdu Instrument Factory). EEG was used to confirm epileptic seizures. Seizures were graded using the Racine scale (20). Seizure latency and duration were also recorded. Rats that survived following $2 \mathrm{~h}$ of continued grade 4 or greater seizures (SE model establishment) were included in the present study. Pentobarbital sodium ( $40 \mathrm{mg} / \mathrm{kg}$; i.p.) was administered to stop seizures. Following SE, rats were kept warm and fed with glucose saline through a gastric tube. Rats in the normal control group $(n=8)$ received sodium chloride ( $0.9 \%$; i.p.) instead of pilocarpine, and rats in the $\mathrm{SE}+$ inhibitor $(\mathrm{SE}+\mathrm{inh})$ group $(\mathrm{n}=6)$ were pretreated with the mTOR inhibitor LY3023414 (10 mg/kg; i.p.) before seizures. Rats were sacrificed with pentobarbital sodium ( $400 \mathrm{mg} / \mathrm{kg}$; i.p.) at $3 \mathrm{~h}$ (SE-3 h group, $\mathrm{n}=9$ ), $6 \mathrm{~h}$ (SE-6 h group, $\mathrm{n}=9$ ), 1 day (SE-1d group, $n=9$ ) and 3 days (SE-3d group, $n=9$ ) following SE.

Histology and immunohistochemistry (IHC). After rats were sacrificed with lethal pentobarbital sodium, the brains were perfused with $0.9 \%$ sodium chloride via intramyocardial injection, in order to excrete blood from the brain of rats. Then, the brains were perfused with $4 \%$ paraformaldehyde at room temperature for $\sim 5 \mathrm{~min}$, after which the brains were harvested and post-fixed in $4 \%$ paraformaldehyde overnight at $4^{\circ} \mathrm{C}$. Brains were embedded in paraffin and sectioned in the coronal plane in $4-\mu \mathrm{m}$ thick slides. The sections containing the hippocampus were selected for IHC. Sections were heated to $98^{\circ} \mathrm{C}$ for 10 min using a microwave in citrate buffer $(\mathrm{pH}, 6.0)$ and treated with $3 \% \mathrm{H}_{2} \mathrm{O}_{2}$ for $15 \mathrm{~min}$ at room temperature to abolish endogenous peroxidase activity. After blocking with 3\% BSA-PBS (cat. no. ST023; Beyotime Institute of Biotechnology) for $30 \mathrm{~min}$ in $37^{\circ} \mathrm{C}$, sections were incubated with the primary antibodies (p-PRAS40; dilution 1:100) at $4^{\circ} \mathrm{C}$ overnight and then with corresponding HRP-conjugated secondary antibodies for $30 \mathrm{~min}$ at $37^{\circ} \mathrm{C}$. The sections were incubated with 3,3-diaminobenzidine solution for $2 \mathrm{~min}$ at room temperature for visualization and were subsequently dehydrated and mounted using neutral balsam. The sections were observed using a light microscope (magnification 400x, Zeiss observer A1; Zeiss AG).

Western blot analysis. After sacrifice, the rat hippocampus was isolated and total proteins were extracted using RIPA buffer (cat. no. P0013; Beyotime Institute of Biotechnology) supplemented with serine protease inhibitor, PMSF. The protein 
A
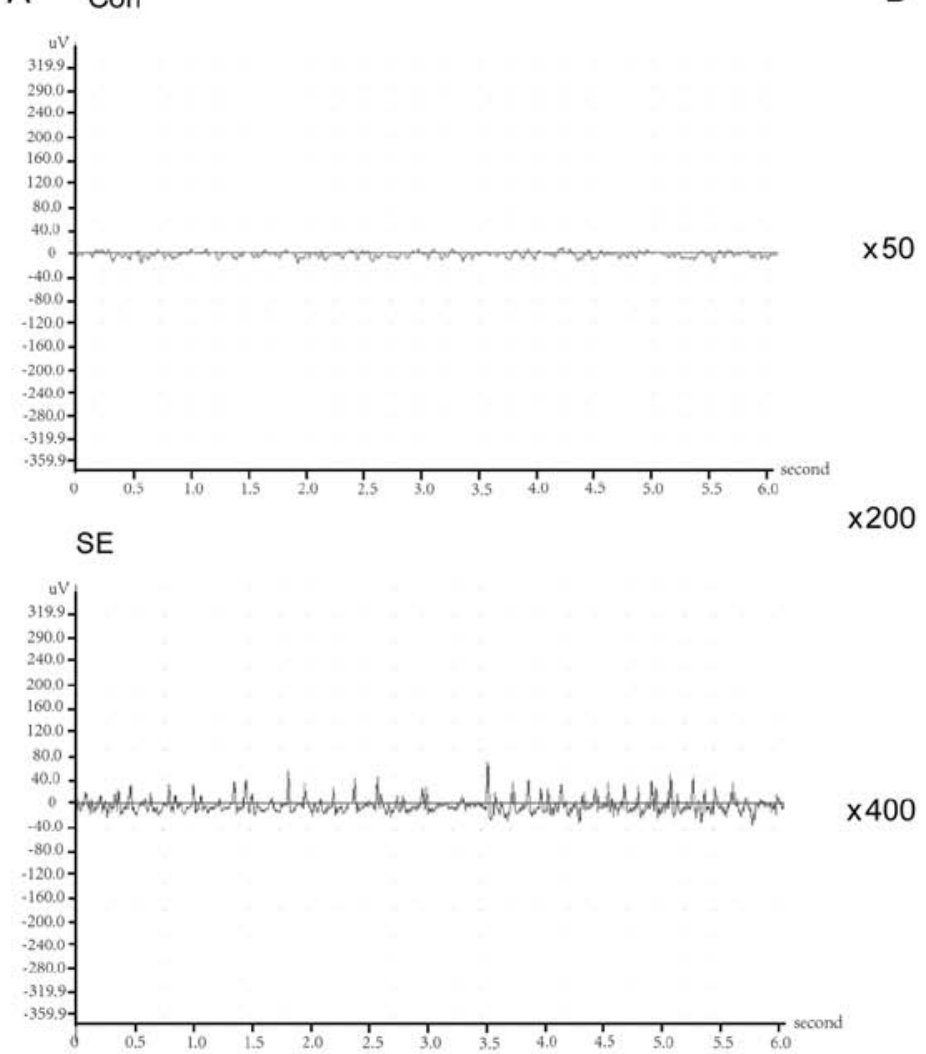

B
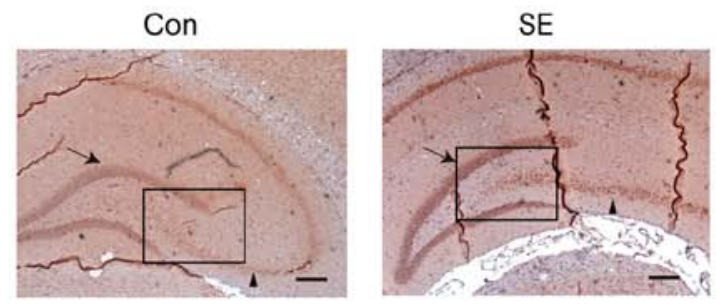

$x 200$
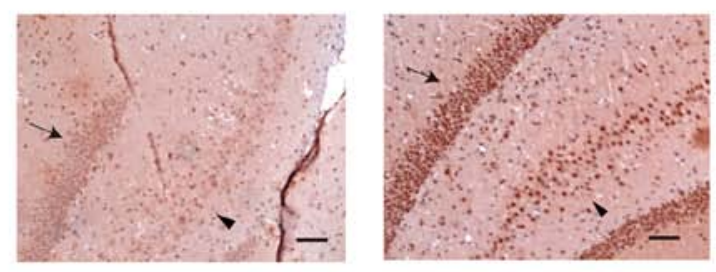

$\times 400$
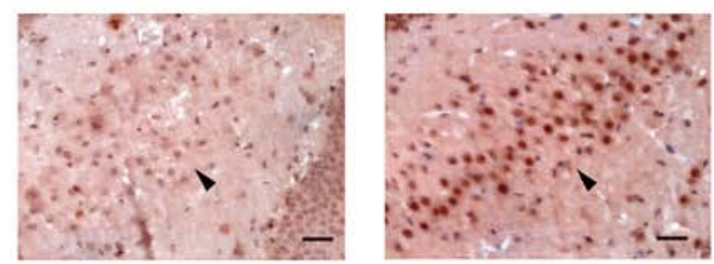

Figure 1. p-PRAS40 is increased in the rat model of SE. (A) Representative EEG of the SE and control rat groups. Compared with the control group, the EEG of the SE groups had more poly-spike waves and a higher wave amplitude. (B) Representative immunohistochemistry images of p-PRAS40 in the control and SE-3 d groups. The figure in the lower row is an enlarged view of the black box. The left label indicates the magnification of each row. There were more p-PRAS40 positive cells in the dentate gyrus (indicated by black arrows pointed) and Cornu Ammonis 3 regions (indicated by triangles) in the SE-3 d group. The positive cell staining of the SE group was greater compared with the control group. d, day; SE, status epilepticus; EEG, electroencephalography; p-, phosphorylated; PRAS40, proline-rich AKT substrate of $40 \mathrm{kDa}$; Con, control.

was measured using a BCA Protein Assay kit (cat. no. P0010; Beyotime Institute of Biotechnology). A total of $30 \mu \mathrm{g}$ protein samples were resolved using $10 \%$ SDS-PAGE, then transferred to PVDF membranes. Then, the membranes were blocked with $5 \%$ non-fat dry milk in Tris-buffered saline/ $0.1 \%$ Tween-20 for $1 \mathrm{~h}$ at $37^{\circ} \mathrm{C}$, followed by incubation with primary antibodies (dilution 1:1,000) at $4^{\circ} \mathrm{C}$ overnight. After $1 \mathrm{~h}$ incubation with the corresponding HRP-conjugated secondary antibodies (dilution 1:1,000) at room temperature, signals were detected using ECL reagents (cat. no. 34096; Thermo Fisher Scientific, Inc.). Signal intensities were quantified using ImageJ v1.28 program (National Institutes of Health) and normalized to GAPDH expression.

Co-immunoprecipitation (Co-IP). Total proteins from the rat hippocampi were extracted for Co-IP using the Pierce Co-IP kit (cat. no. 26149; Thermo Fisher Scientific, Inc.) according to manufacturer's protocol. Briefly, 50\% AminoLink resin was added into a Pierce Spin Column at room temperature. Primary antibodies (p-PRAS40; dilution 1:10) were added into the resin in the spin column and incubated on a rotator at room temperature for $120 \mathrm{~min}$. Subsequently, the total protein lysates, collected using IP Lysis Buffer, following the protocol of this kit, of experimental group and the controls (including the negative control and the blank control) were added to the resin and incubated with gentle rocking overnight at $4^{\circ} \mathrm{C}$. The negative control used IgG instead of the p-PRAS40 antibody. Positive control was used as the whole control protein to immunoblot directly. The eluent was subjected to $12 \%$ SDS-PAGE followed by western blot analysis using the 14-3-3 antibody (dilution 1:500).

Statistical analysis. Continuous variables were presented as the mean \pm SD. Each experiment was repeated at least three times. Data were analyzed using SPSS 17.0 (SPSS, Inc.) and bar graphs were created using GraphPad Prism (version 5.0; GraphPad Software, Inc.). If the data distribution was normal, comparisons among $\geq 3$ groups were calculated with a one-way ANOVA test followed by Tukey's test. If the data distribution was not normal, comparisons were calculated using Kruskal-Wallis followed by Dunn's test for non-parametric analysis. $\mathrm{P}<0.05$ was considered to indicate a statistically significant difference.

\section{Results}

p-PRAS40 levels are elevated following SE. First, the present study established a pilocarpine-induced rat model to simulate SE. The EEG identified that the SE group produced high amplitudes (between 100-200 $\mu \mathrm{V}$ ) with superimposed, mildly fast and poly-spike waves in the $\theta$ range. However, the control group was recorded with mildly synchronous and well-organized waves that were in the normal amplitude range 
A

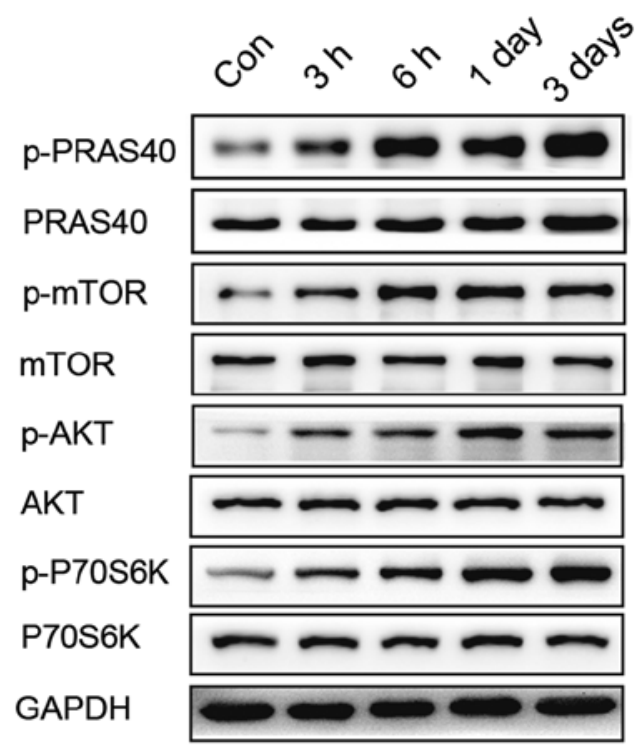

C IP : p-PRAS40

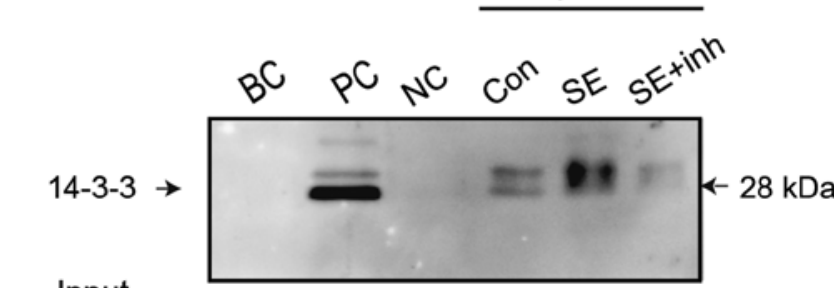

Input GAPDH

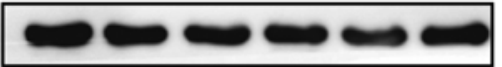

D

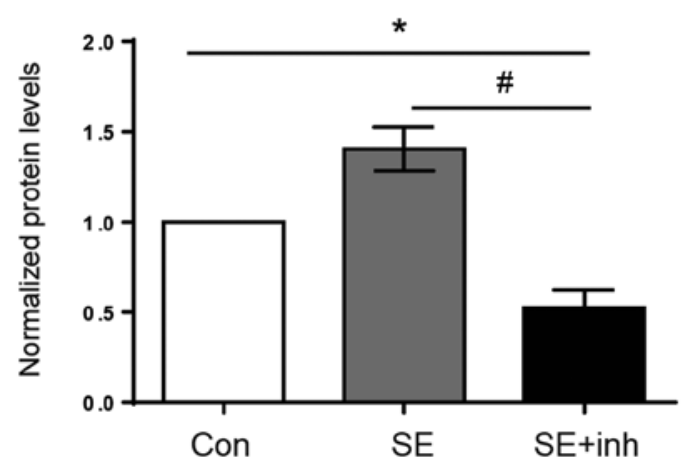

B
p-PRAS40

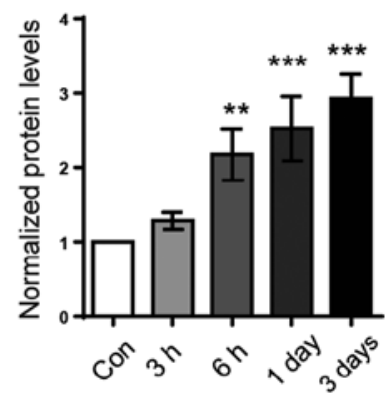

p-PRAS40/PRAS40

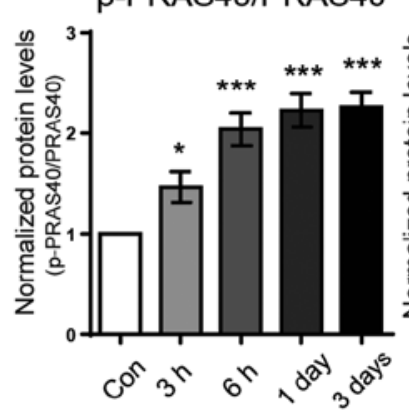

PRAS40
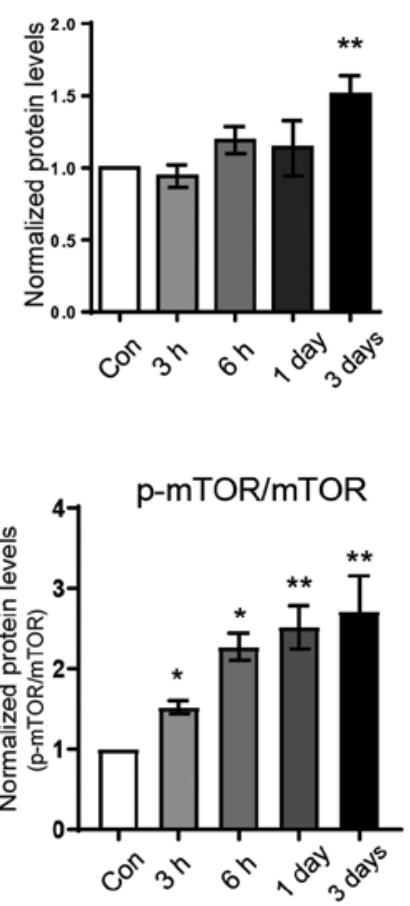

p-AKT/AKT

p-P70S6K/P70S6K

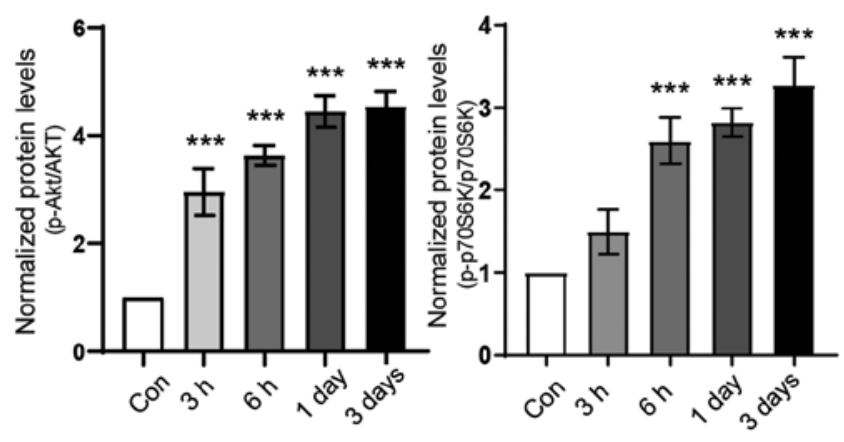

Figure 2. p-PRAS40 combines with 14-3-3 protein to activate the mTOR signaling pathway. (A) Western blot analysis of p-PRAS40, PRAS40, p-mTOR, mTOR, p-AKT, AKT, p-P70S6K and P70S6K expression levels in the rat hippocampus following SE at the indicated times. (B) Representative proteins of the PI3K/AKT/mTOR signaling pathway were increased with the upregulation of p-PRAS40 (n=4 per group). (C) Proteins from the control, SE and SE + inh groups were immunoprecipitated with p-PRAS40 and immunoblotted with the 14-3-3 antibody. The NC used IgG instead of the p-PRAS40 antibody. PC was used as the whole control protein to immunoblot directly. (D) Co-IP analysis demonstrated that the combination of p-PRAS40 with 14-3-3 protein was enhanced following SE and the inhibition of p-AKT significantly suppressed the combination of these two proteins. Data were analyzed using ANOVA ( $\mathrm{n}=3$ ). ${ }^{*} \mathrm{P}<0.05,{ }^{* *} \mathrm{P}<0.01$ and ${ }^{* * *} \mathrm{P}<0.001$ vs. control; ${ }^{\#} \mathrm{P}<0.05$ vs. SE group. NC, negative control; PC, positive control, which is the whole lysate; BC, Blank control; Co-IP, Co-immunoprecipitation; SE, status epilepticus; p-, phosphorylated; PRAS40, proline-rich AKT substrate of 40 kDa; Con, control; inh, inhibitor; P70S6K, ribosomal protein S6 kinase 1.

(between 5-50 $\mu \mathrm{V}$ ). In addition, the control showed no focal sharp or spike wave activities (Fig. 1A).

To investigate whether the expression of p-PRAS40 was elevated in the rat hippocampus following SE, IHC was used to detect the expression of p-PRAS40. Compared with the control group, p-PRAS40 positive cells in the dentate gyrus and Cornu Ammonis (CA) 3 regions of the hippocampus were markedly increased in the SE-3d group (Fig. 1B).
The western blot analysis results suggested that p-PRAS40 expression was significantly upregulated at the indicated times following seizures, while the expression of PRAS40 was slightly increased. Quantification of the ratio of p-PRAS40 and PRAS40 demonstrated a significant increase in a time-dependent manner (Fig. 2A and B). These results indicated that p-PRAS40 was significantly elevated over time following acute SE. 
A

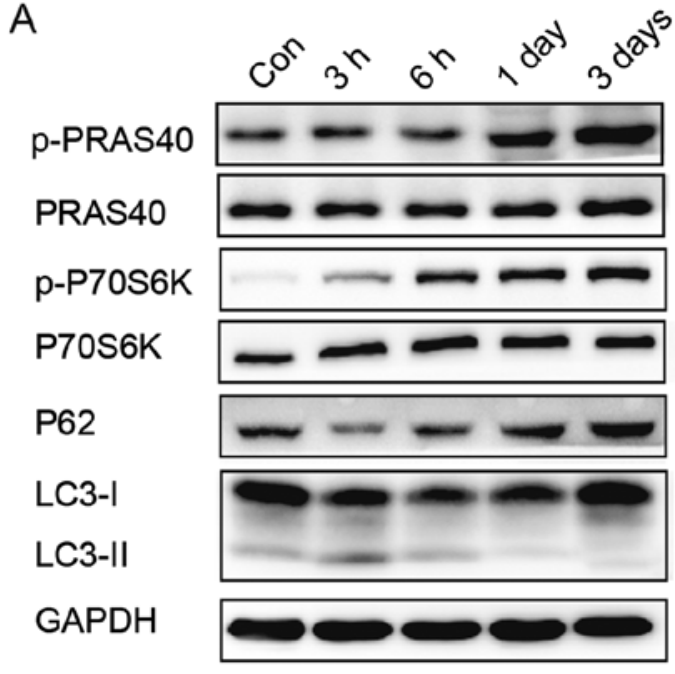

B
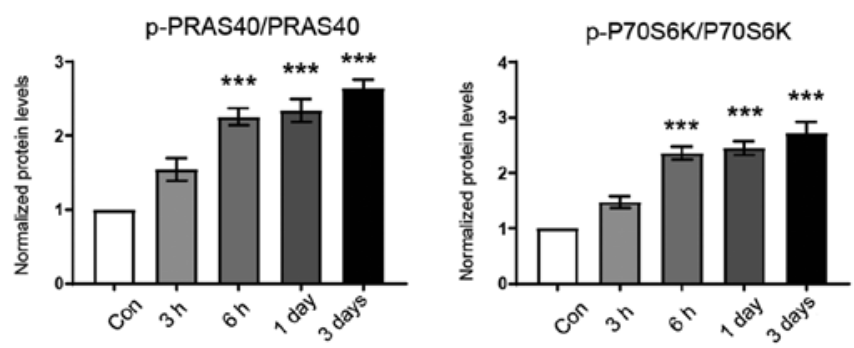

LC3-II/LC3-I
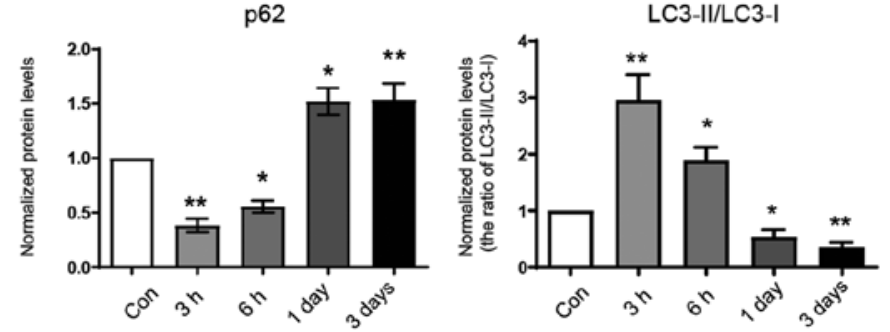

C

D

p-mTOR/mTOR
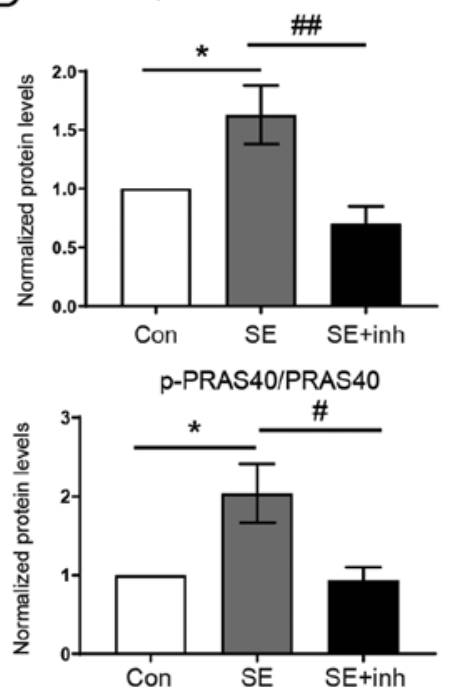

P62 \#

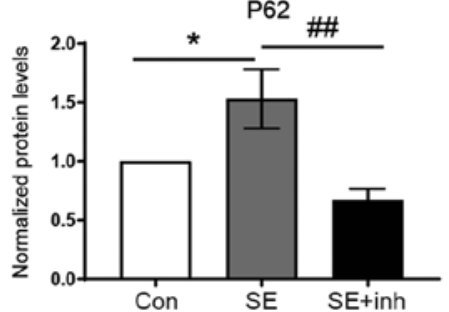

p-AKT/AKT
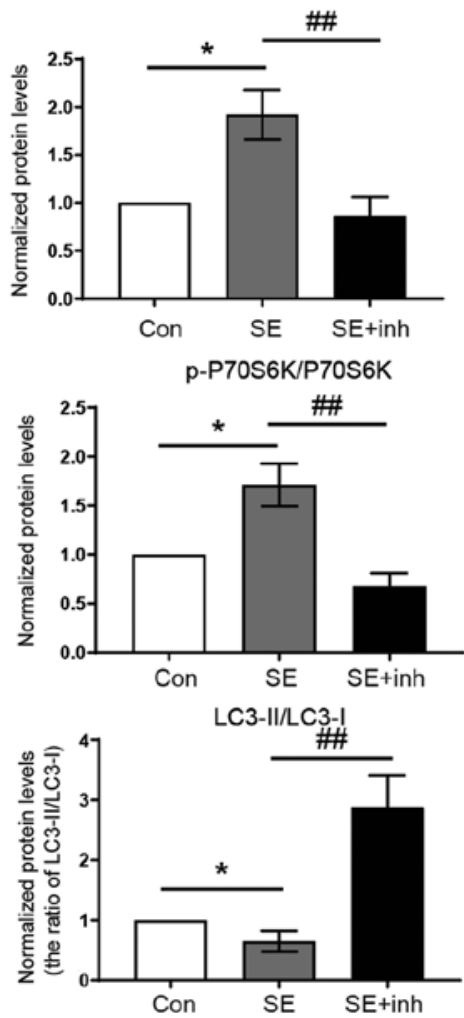

Figure 3. Increased p-PRAS40 expression activates the mTOR signaling pathway to suppress autophagy. (A) Protein expression levels of p-PRAS40, PRAS40, p-P70S6K, P70S6K, P62 and LC3 was detected by western blotting. (B) p-PRAS40 and p-P70S6K expression levels were increased following SE at the indicated times. However, the autophagy markers LC3 and P62 were decreased over time (n=4 per group). (C) Western blot analysis of the PI3K/AKT/mTOR signaling pathway expression and autophagy levels in the SE group and the SE + inh group, when the p-AKT pathway was inhibited using LY3023414. (D) After p-AKT was inhibited by LY3023414 in the SE + inh group, the autophagy level was increased with the decreased levels of p-PRAS40 and AKT/mTOR pathway-associated proteins. Data were analyzed using ANOVA $(\mathrm{n}=4)$. ${ }^{*} \mathrm{P}<0.05,{ }^{* *} \mathrm{P}<0.01$ and ${ }^{* * *} \mathrm{P}<0.001$ vs. control; ${ }^{*} \mathrm{P}<0.05$ and ${ }^{\# \#} \mathrm{P}<0.01$ vs. SE group. SE, status epilepticus; p-, phosphorylated; PRAS40, proline-rich AKT substrate of $40 \mathrm{kDa}$; Con, control; inh, inhibitor; P70S6K, ribosomal protein S6 kinase 1; LC3, light chain 3.

p-PRAS40 activates the PI3K/AKT/mTOR pathway via combination with the 14-3-3 scaffold protein. Since PRAS40 is downstream of p-AKT, and the phosphorylation of PRAS40 can lead to the activation of the mTOR pathway (21), PI3K/AKT/mTOR pathway activation was detected in the present study. The protein expression levels of p-mTOR, p-AKT,
p-P70S6K and p-PRAS40 in all of the experimental groups were significantly higher compared with the control group, and were increased in a time-dependent manner (Fig. 2A and B).

The combination of p-PRAS40 and 14-3-3 scaffold protein leads to the activation of the mTOR pathway (21), and thus Co-IP was used to detect this combination. It was found that 
p-PRAS40/14-3-3 was markedly increased 3 days following SE compared with the control group (Fig. 2C and D). Moreover, LY3023414, the inhibitor of AKT phosphorylation, significantly reduced the binding of p-PRAS40 and 14-3-3 scaffold protein compares with the SE group (Fig. 2C and D).

Increased expression of $p$-PRAS40 is associated with reduced levels of autophagy flux. The mTOR pathway inhibits the initiation of autophagy and the phosphorylation of PRAS40 regulates mTOR activity $(5,14)$; therefore, the current study detected alterations in p-PRAS40 expression, mTOR activity and autophagy flux following SE. The increase in the ratio of LC3-II to LC3-I and the decrease in P62 expression levels indicated that the levels of autophagy were significantly elevated $3 \mathrm{~h}$ following SE (Fig. 3A and B). However, with the increase of p-PRAS40 and the activation of the mTOR pathway, the autophagy levels decreased in a time-dependent manner.

To confirm the p-PRAS40 could regulate the autophagy, LY3023414 was used to inhibit the PI3K/AKT signaling pathway and p-PRAS40 in the SE + inh group. As shown in Fig. 3C, after the inhibition of p-PRAS40, the expression levels of components of the mTOR pathway were decreased compared the SE groups, including p-mTOR and p-P70S6K. The rate of conversion of LC3-II to LC3-I was increased, the expression levels of P62 were decreased, which indicated that the levels of autophagy in the SE + inh group were significantly higher compared to the SE groups These results suggested that p-PRAS40 may regulate the autophagy flux.

\section{Discussion}

The mTOR pathway is important in the epileptogenic mechanism. Moreover, PRAS40 is a subunit of mTORC1 and p-PRAS40 can regulate mTOR activity (21). However, to the best of our knowledge, there are few reports examining the relationship between PRAS40 and SE. The present results suggested that p-PRAS40 expression was abnormally elevated following SE. In addition, the IHC staining identified that p-PRAS40 positive cells increased significantly in the rat hippocampal dentate gyrus and CA3 region 3 days following SE. Western blotting results also demonstrated this effect and indicated an increasing trend over time, which suggested that p-PRAS40 was increased continuously after SE.

The dentate gyrus is composed of granular cells, whose axons are covered in mossy fibers that project into the CA3 region (22). Pyramidal cells in the CA3 region are reported to be more active during epileptic activity than in other brain areas (22). Furthermore, mossy-fiber sprouting (MFS) as a result of damage to pyramidal cells in the CA3 region, the rupture of mossy fibers and abnormal synaptic connections between lateral growing axons and granular cells in the dentate gyrus, is the pathological basis of chronic temporal epilepsy (23). The present results indicated that p-PRAS40 may participate in the formation of chronic epileptogenic focus; however, this requires further investigation. IHC staining demonstrated that the vast majority of p-PRAS40 existed in neuronal nuclei, which was consistent with previous reports that p-PRAS40 (Thr246) is mainly located in nucleus (24-26).

PRAS40 is both the substrate of the PI3K/AKT pathway and the subunit of mTORC1 (5). Therefore, it was hypothesized that PRAS40 may be the key link between the PI3K/AKT and mTOR pathways following SE. The western blotting results suggested that p-PRAS40 expression was elevated following SE, and the expression of upstream PI3K and p-AKT, as well as the p- of downstream P70S6K were increased. P70S6K is a substrate of mTORC1 and p-P70S6K is considered to be a reliable marker of mTOR activation (27). Thus, it could be speculated that following SE, activation of the PI3K/AKT pathway promoted p-PRAS40, which in turn increased the activity of the mTOR pathway. In addition, the current findings demonstrated that the increasing expression levels of PI3K, p-AKT and p-P70S6K were similar to that of p-PRAS40 at different time points following SE, which could also indicate that PRAS40 was associated with the PI3K/AKT and mTOR pathways following SE.

p-PRAS40 binds to the 14-3-3 scaffold protein and then separates from mTORC1 (6). In the present study, Co-IP was used to detect the status of this combination. It was found that the levels of p-PRAS40 combined with 14-3-3 were higher in the SE group compared with the control group, but the difference was not statistically significant. Nevertheless, this combination could be significantly inhibited by LY3023414, an inhibitor of PI3K, which may partially attenuate the prior defects. Both PI3K/AKT/PRAS40/mTOR pathway activation and increasing the expression of p-PRAS40 combined with 14-3-3 were detected following SE, and therefore it was speculated that the interaction of p-PRAS40 with 14-3-3 may contribute to the dissociation of p-PRAS40 with mTORC1 and the activation of the mTOR pathway.

Autophagy is a double-edged sword. Under normal physiological conditions, autophagy can help to promote cell survival and maintain homeostasis by eliminating damaged cell organelles and toxic metabolites (28). However, excessive autophagy can accelerate cell death, which is associated with disease development (18). Previous studies have reported insufficient autophagy in chronic neurological diseases, such as Parkinson's disease (29), Alzheimer's disease (30) and Lafora's disease (31). Moreover, autophagy contributes to the elimination of abnormal protein aggregates in cells and may provide protection against disease (13), and there has been a similar report in chronic epilepsy. Hosseinzadeh et al (32) revealed that cannabidiol could enhance the induction of the autophagy pathway as a protective mechanism in temporal epilepsy. Furthermore, Ni et al (33) reported that insufficient autophagy existed in neonatal rats with chronic epilepsy, which exhibited consistent trends with rat hippocampal MFS and cognitive deficits. However, an abnormal increase in autophagy was demonstrated in acute neurological disorders such as encephalitis (34) and cerebral infarction (35), which may be associated with acute neurocyte death. Controversy remains regarding how autophagy changes after SE. For instance, previous studies have suggested that the autophagy level increase following SE $(15,16)$, but others have indicated that autophagy after SE has a dynamic course (17-19). Since mTOR is one of major pathways regulating autophagy that can inhibit autophagy initiation, it was hypothesized that PRAS40 may participate in the regulation of autophagy by influencing mTOR activity.

LC3 is an important protein involved in autophagosome formation (36). Furthermore, LC3 participates in the elongation of bilayer bio-membranes, cytoplasm and defective protein, as well as autophagosome formation (37). In the cytoplasm, LC3 exists as LC3-I. When autophagosomes begin to form, LC3-I transforms to LC3-II, which binds to the autophagosome 
membrane (38). Therefore, the ratio of LC3-II/LC3-I was used in the present study to represent autophagy level. P62 was first reported as a polyubiquitin-binding protein in autophagy (39). It has been shown that P62 can bind to ubiquitinated protein and produce ubiquitinated protein aggregates, which serve an essential role in the degradation of ubiquitinated proteins in autophagosomes (40). Thus, P62 expression is the opposite to that of autophagy degradation, that is, the greater the levels of degradation, the lower the P62 expression (41). Therefore, increasing LC3-II/LC3-I levels and decreasing levels of P62 occurring concurrently suggest that the autophagy flux is unobstructed. Thus, LC3-II/LC3-I and P62 were detected in the present study to evaluate the autophagy flux.

In the current study, p-PRAS40 expression, mTOR pathway activity and autophagy flux were detected at various time points following SE. Autophagy levels were significantly elevated at $3 \mathrm{~h}$ post-SE, and gradually decreased over time. In addition, p-PRAS40 expression was increased and the mTOR pathway was found to be more active, with a prolonged duration of SE. Thus, it was speculated that autophagy was abnormally elevated due to various factors, such as inflammation and oxidative stress, occurring shortly following SE induction, which then gradually decreased with increased p-PRAS40 levels and mTOR activation. Since an experimental group earlier than $3 \mathrm{~h}$ post-SE was not used in the current study, the mechanism underlying the increase in autophagy was not observed, which was a limitation of the study design. However, to investigate the aforementioned hypothesis, a LY3023414 pretreated SE group was established at 3 days after SE (highest expression of p-PRAS40 and lowest autophagy level) and the $\mathrm{PI} 3 \mathrm{~K} / \mathrm{AKT} / \mathrm{PRAS} 40 / \mathrm{mTOR}$ pathway and autophagy flux were detected in the control, SE-3d and SE-3d + inh groups. The results demonstrated that there was abnormal activation of the PI3K/AKT/PRAS40/mTOR pathway and decreased autophagy in the SE-3d group, and that LY3023414 pretreatment could inhibit the activation of this pathway and increase the levels of autophagy, which suggested that p-PRAS40 participated in the mTOR-associated downregulation of autophagy.

Previous studies have reported that the abnormal elevation of autophagy following SE may be associated with neurocyte death $(15,16)$. Therefore, it was hypothesized that p-PRAS40 and mTOR activation may have a protective effect on neurocyte after SE by inhibiting autophagy. However, abnormal activation of the mTOR pathway participates in chronic epilepsy development (42), and thus it was speculated that long-term high expression of p-PRAS40 and activation of the mTOR pathway may be associated with epileptic foci formation, which should be further investigated in future research.

In conclusion, to the best of our knowledge, the present study was the first to identify the role of p-PRAS40 and its associated mechanism contributing to SE pathogenesis. Currently, the available antiepileptic drugs suppress seizures and are used as a symptomatic therapy (1). Thus, these drugs are a short-term medication that can prevent the development of epilepsy. The present results may represent a novel and promising therapeutic target with antiepileptogenic effects. Therefore, further studies should focus on PRAS40 function in other types of epilepsy, such as temporal lobe epilepsy, as well as investigating treatments that target p-PRAS40.

\section{Acknowledgements}

The authors would like to thank the support from technician Mrs. Jieqing Jiang at the Department of Forensic Medicine, Fudan University.

\section{Funding}

This study was supported by Shanghai Key Laboratory of Crime Scene Evidence, Institute of Forensic Science, Shanghai Public Security Bureau (grant nos. 2015XCWZK01 and 2017XCWZK170), Funds for Youth in Department of Forensic Medicine, School of Basic Medical Sciences, Fudan University (grant no. FY2014-01), Sponsored by the Medical Scientific Research Project of Xuhui District, 2018 (grant no. SHXH201818) and Remote Forensic Consultation Center, Collaborative Innovation Center of Judicial Civilization, China University of Political Science and Law (grant no. XUF101010). The funders had no role in study design, data collection and analysis, decision to publish or preparation of the manuscript.

\section{Availability of data and materials}

The datasets used and/or analyzed during the current study are available from the corresponding author on reasonable request.

\section{Authors' contributions}

YS and BL designed the study, and analyzed and interpreted the data. JL, YF and MZ contributed to and conducted the experiments and contributed to writing the manuscript. $\mathrm{XW}, \mathrm{LL}, \mathrm{MH}, \mathrm{AX}$ and $\mathrm{KZ}$ made substantial contributions to the experiments, the acquisition of data, interpretation of experiments and revised the manuscript. All authors read and approved the final manuscript.

\section{Ethics approval and consent to participate}

The present study was approved by the Experimental Animal Ethics Committee of the Basic Medical College of Fudan University.

\section{Patient consent for publication}

Not applicable.

\section{Competing interest}

The authors declare that they have no competing interests.

\section{References}

1. Betjemann JP and Lowenstein DH: Status epilepticus in adults. Lancet Neurol 14: 615-624, 2015.

2. Trinka E, Cock H, Hesdorffer D, Rossetti AO, Scheffer IE, Shinnar S, Shorvon S and Lowenstein DH: A definition and classification of status epilepticus-report of the ILAE task force on classification of status epilepticus. Epilepsia 56: 1515-1523, 2015.

3. Wong M: Mammalian target of rapamycin (mTOR) inhibition as a potential antiepileptogenic therapy: From tuberous sclerosis to common acquired epilepsies. Epilepsia 51: 27-36, 2010. 
4. Kovacina KS, Park GY, Bae SS, Guzzetta AW, Schaefer E, Birnbaum MJ and Roth RA: Identification of a proline-rich Akt substrate as a 14-3-3 binding partner. J Biol Chem 278: 10189-10194, 2003.

5. Nascimento EB, Snel M, Guigas B, van der Zon GCM, Kriek J, Maassen JA, Jazet IM, Diamant M and Ouwens DM: Phosphorylation of PRAS40 on Thr246 by PKB/AKT facilitates efficient phosphorylation of Ser183 by mTORC1. Cell Signal 22: 961-967, 2010

6. Thedieck K, Polak P, Kim ML, Molle KD, Cohen A, Jenö P, Arrieumerlou C and Hall MN: PRAS40 and PRR5-like protein are new mTOR interactors that regulate apoptosis. PLoS One 2: e1217, 2007.

7. Kazi AA and Lang CH: PRAS40 regulates protein synthesis and cell cycle in C2C12 myoblasts. Mol Med 16: 359-371, 2010.

8. Hong-Brown LQ, Brown CR, Kazi AA, Huber DS, Pruznak AM and Lang $\mathrm{CH}$ : Alcohol and PRAS40 knockdown decrease mTOR activity and protein synthesis via AMPK signaling and changes in mTORC1 interaction. J Cell Biochem 109: 1172-1184, 2010.

9. Lipton JO and Sahin M: The neurology of mTOR. Neuron 84 275-291, 2014

10. San YZ, Liu Y, Zhang Y, Shi PP and Zhu YL: Peroxisome proliferator-activated receptor- $\gamma$ agonist inhibits the mammalian target of rapamycin signaling pathway and has a protective effect in a rat model of status epilepticus. Mol Med Rep 12: 1877-1883, 2015.

11. Brewster AL, Lugo JN, Patil VV, Lee WL, Qian Y, Vanegas F and Anderson AE: Rapamycin reverses status epilepticus-induced memory deficits and dendritic damage. PLoS One 8: e57808, 2013.

12. Wang SJ, Bo QY, Zhao XH, Yang X, Chi ZF and Liu XW: Resveratrol pre-treatment reduces early inflammatory responses induced by status epilepticus via mTOR signaling. Brain Res 1492: 122-129, 2013.

13. Kim KH and Lee MS: Autophagy-a key player in cellular and body metabolism. Nat Rev Endocrinol 10: 322-337, 2014.

14. Munson MJ and Ganley IG: MTOR, PIK3C3, and autophagy: Signaling the beginning from the end. Autophagy 11: 2375-2376, 2015.

15. Cao L, Xu J, Lin Y, Zhao X, Liu X and Chi Z: Autophagy is upregulated in rats with status epilepticus and partly inhibited by vitamin E. Biochem Biophys Res Commun 379: 949-953, 2009.

16. Dong Y, Wang S, Zhang T, Zhao X, Liu X, Cao L and Chi Z: Ascorbic acid ameliorates seizures and brain damage in rats through inhibiting autophagy. Brain Res 1535: 115-123, 2013

17. Benz AP, Niquet J, Wasterlain CG and Rami A: Status epilepticus in the immature rodent brain alters the dynamics of autophagy. Curr Neurovasc Res 11: 125-135, 2014.

18. Rami A, Benz AP, Niquet J and Langhagen A: Axonal accumulation of lysosomal-associated membrane protein 1 (LAMP1) accompanying alterations of autophagy dynamics in the rat hippocampus upon seizure-induced injury. Neurochem Res 41 : 53-63, 2016.

19. Shacka JJ, Lu J, Xie ZL, Uchiyama Y, Roth KA and Zhang J: Kainic acid induces early and transient autophagic stress in mouse hippocampus. Neurosci Lett 414: 57-60, 2007.

20. Racine RJ: Modification of seizure activity by electrical stimulation. II. Motor seizure. Electroencephalogr Clin Neurophysiol 32: 281-294, 1972

21. Dibble CC and Cantley LC: Regulation of mTORC1 by PI3K signaling. Trends Cell Biol 25: 545-555, 2015.

22. Sloviter RS: The functional organization of the hippocampal dentate gyrus and its relevance to the pathogenesis of temporal lobe epilepsy. Ann Neurol 35: 640-654, 1994.

23. Sutula TP and Dudek FE: Unmasking recurrent excitation generated by mossy fiber sprouting in the epileptic dentate gyrus: An emergent property of a complex system. Prog Brain Res 163: 541-563, 2007.

24. Nascimento EB, Fodor M, van der Zon GC, Jazet IM, Meinders AE, Voshol PJ, Vlasblom R, Baan B, Eckel J, Maassen JA, et al: Insulin-mediated phosphorylation of the proline-rich Akt substrate PRAS40 is impaired in insulin target tissues of high-fat diet-fed rats. Diabetes 55: 3221-3228, 2006.

25. Saito A, Hayashi T, Okuno S, Nishi T and Chan PH: Modulation of proline-rich akt substrate survival signaling pathways by oxidative stress in mouse brains after transient focal cerebral ischemia. Stroke 37: 513-517, 2006.
26. Saito A, Narasimhan P, Hayashi T, Okuno S, Ferrand-Drake M and Chan PH: Neuroprotective role of a proline-rich Akt substrate in apoptotic neuronal cell death after stroke: Relationships with nerve growth factor. J Neurosci 24: 1584-1593, 2004.

27. Feliciano DM, Su T, Lopez J, Platel JC and Bordey A: Single-cell Tsc1 knockout during corticogenesis generates tuber-like lesions and reduces seizure threshold in mice. J Clin Invest 121: 1596-1607, 2011

28. Luo CL, Li BX, Li QQ, Chen XP, Sun YX, Bao HJ, Dai DK, Shen YW, Xu HF, Ni H, et al: Autophagy is involved in traumatic brain injury-induced cell death and contributes to functional outcome deficits in mice. Neuroscience 184: 54-63, 2011.

29. Wang K, Huang J, Xie W, Huang L, Zhong C and Chen Z: Beclin1 and HMGB1 ameliorate the $\alpha$-synuclein-mediated autophagy inhibition in PC12 cells. Diagn Pathol 11: 15, 2016.

30. Shin JY, Park HJ, Kim HN, Oh SH, Bae JS, Ha HJ and Lee PH: Mesenchymal stem cells enhance autophagy and increase $\beta$-amyloid clearance in Alzheimer disease models. Autophagy 10: $32-44,2014$.

31. Aguado C, Sarkar S, Korolchuk VI, Criado O, Vernia S, Boya P, Sanz P, de Córdoba SR, Knecht E and Rubinsztein DC: Laforin, the most common protein mutated in Lafora disease, regulates autophagy. Hum Mol Genet 19: 2867-2876, 2010.

32. Hosseinzadeh M, Nikseresht S, Khodagholi F, Naderi N and Maghsoudi N: Cannabidiol post-treatment alleviates rat epileptic-related behaviors and activates hippocampal cell autophagy pathway along with antioxidant defense in chronic phase of pilocarpine-induced seizure. J Mol Neurosci 58: 432-440, 2016

33. Ni H, Zhao DJ and Tian T: Ketogenic diet change cPLA2/clusterin and autophagy related gene expression and correlate with cognitive deficits and hippocampal MFs sprouting following neonatal seizures. Epilepsy Res 120: 13-18, 2016.

34. Jin R, Zhu W, Cao S, Chen R, Jin H, Liu Y, Wang S, Wang W and Xiao G: Japanese encephalitis virus activates autophagy as a viral immune evasion strategy. PLoS One 8: e52909, 2013.

35. Li L, Tian J, Long MK, Chen Y, Lu J, Zhou C and Wang T: Protection against experimental stroke by ganglioside GM1 is associated with the inhibition of autophagy. PLoS One 11: e144219, 2016.

36. Klionsky DJ, Abdalla FC, Abeliovich H, Abraham RT, Acevedo-Arozena A, Adeli K, Agholme L, Agnello M, Agostinis P, Aguirre-Ghiso JA, et al: Guidelines for the use and interpretation of assays for monitoring autophagy. Autophagy 8: 445-544, 2012.

37. Schaaf MB, Keulers TG, Vooijs MA and Rouschop KM: LC3/GABARAP family proteins: Autophagy-(un)related functions. FASEB J 30: 3961-3978, 2016.

38. Sini P, James D, Chresta C and Guichard S: Simultaneous inhibition of mTORC1 and mTORC2 by mTOR kinase inhibitor AZD8055 induces autophagy and cell death in cancer cells. Autophagy 6: 553-554, 2010.

39. Lamark T, Svenning S and Johansen T: Regulation of selective autophagy: The p62/SQSTM1 paradigm. Essays Biochem 61: 609-624, 2017.

40. Nezis IP, Simonsen A, Sagona AP, Finley K, Gaumer S, Contamine D, Rusten TE, Stenmark H and Brech A: Ref(2)P, the drosophila melanogaster homologue of mammalian p62, is required for the formation of protein aggregates in adult brain. J Cell Biol 180: 1065-1071, 2008.

41. Bartlett BJ, Isakson P, Lewerenz J, Sanchez H, Kotzebue RW, Cumming RC, Harris GL, Nezis IP, Schubert DR, Simonsen A and Finley KD: p62, ref(2)P and ubiquitinated proteins are conserved markers of neuronal aging, aggregate formation and progressive autophagic defects. Autophagy 7: 572-583, 2011.

42. Buckmaster PS, Ingram EA and Wen X: Inhibition of the mammalian target of rapamycin signaling pathway suppresses dentate granule cell axon sprouting in a rodent model of temporal lobe epilepsy. J Neurosci 29: 8259-8269, 2009.

This work is licensed under a Creative Commons Attribution-NonCommercial-NoDerivatives 4.0 International (CC BY-NC-ND 4.0) License. 